
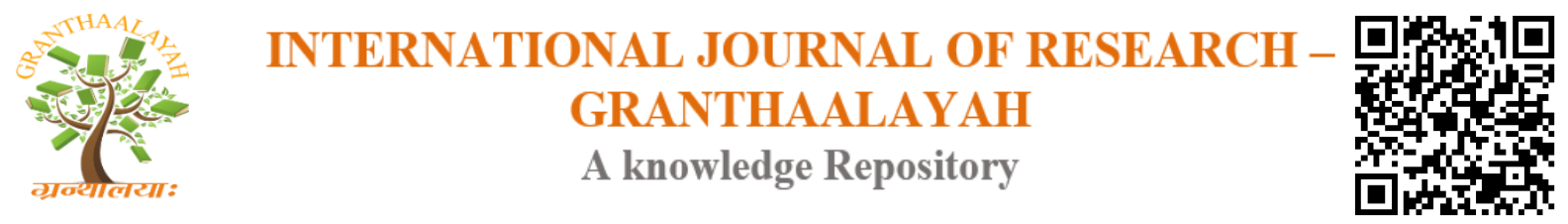

Science

\title{
TRUE POWER LOSS REDUCTION BY HARMONY SEARCH ALGORITHM
}

\author{
Dr. K. Lenin *1 \\ ${ }^{* 1}$ Professor, Department of EEE, Prasad V.Potluri Siddhartha Institute of Technology, Kanuru, \\ Vijayawada, Andhra Pradesh -520007, India
}

\begin{abstract}
This paper presents Harmony Search algorithm (HS) for solving the reactive power problem. Real power loss minimization is the major objective $\&$ also voltage profiles are should be kept within the limits. This paper introduces a new search model the harmony search (HS) algorithm is a relatively new population-based metaheuristic optimization algorithm. It emulates the music improvisation progression where musicians improvise their instruments' pitch by searching for a perfect state of harmony. In order to evaluate the efficiency of the proposed algorithm, it has been tested on practical 191 test system \& real power loss has been considerably reduced.
\end{abstract}

Keywords: Optimal Reactive Power; Transmission Loss; Harmony Search; Optimization.

Cite This Article: Dr. K. Lenin. (2018). “TRUE POWER LOSS REDUCTION BY HARMONY SEARCH ALGORITHM.” International Journal of Research - Granthaalayah, 6(9), 196-205. https://doi.org/10.5281/zenodo.1436806.

\section{Introduction}

Main objective of optimal reactive power problem is to minimize the real power loss. Various numerical methods like the gradient method [1-2], Newton method [3] and linear programming [4-7] have been adopted to solve the optimal reactive power dispatch problem. Both the gradient and Newton methods have the complexity in managing inequality constraints. If linear programming is applied then the input- output function has to be uttered as a set of linear functions which mostly lead to loss of accuracy. The problem of voltage stability and collapse play a major role in power system planning and operation [8]. Evolutionary algorithms such as genetic algorithm have been already proposed to solve the reactive power flow problem [9-11]. Evolutionary algorithm is a heuristic approach used for minimization problems by utilizing nonlinear and non-differentiable continuous space functions. In [12], Hybrid differential evolution algorithm is proposed to improve the voltage stability index. In [13] Biogeography Based algorithm is projected to solve the reactive power dispatch problem. In [14], a fuzzy based method is used to solve the optimal reactive power scheduling method. In [15], an improved evolutionary programming is used to solve the optimal reactive power dispatch problem. In [16], the optimal reactive power flow problem is solved by integrating a genetic algorithm with a nonlinear interior 
point method. In [17], a pattern algorithm is used to solve ac-dc optimal reactive power flow model with the generator capability limits. In [18], F. Capitanescu proposes a two-step approach to evaluate Reactive power reserves with respect to operating constraints and voltage stability. In [19], a programming based approach is used to solve the optimal reactive power dispatch problem. In [20], A. Kargarian et al present a probabilistic algorithm for optimal reactive power provision in hybrid electricity markets with uncertain loads. This paper introduces a new search model the harmony search (HS) algorithm [21-24] is a relatively new population-based metaheuristic optimization algorithm. It emulates the music improvisation progression where musicians improvise their instruments' pitch by searching for a perfect state of harmony. In order to evaluate the efficiency of proposed algorithm, it has been tested on practical 191 test system \& real power loss has been considerably reduced.

\section{Problem Formulation}

The optimal power flow problem is treated as a general minimization problem with constraints, and can be mathematically written in the following form:

Minimize $f(x, u)$

subject to $\mathrm{g}(\mathrm{x}, \mathrm{u})=0$

and

$\mathrm{h}(\mathrm{x}, \mathrm{u}) \leq 0$

Where $f(x, u)$ is the objective function. $g(x . u)$ and $h(x, u)$ are respectively the set of equality and inequality constraints. $\mathrm{x}$ is the vector of state variables, and $\mathrm{u}$ is the vector of control variables. The state variables are the load buses (PQ buses) voltages, angles, the generator reactive powers and the slack active generator power:

$\mathrm{x}=\left(\mathrm{P}_{\mathrm{g} 1}, \theta_{2}, \ldots, \theta_{\mathrm{N}}, \mathrm{V}_{\mathrm{L} 1}, ., \mathrm{V}_{\mathrm{LNL}}, \mathrm{Q}_{\mathrm{g} 1}, \ldots, \mathrm{Q}_{\mathrm{gng}}\right)^{\mathrm{T}}$

The control variables are the generator bus voltages, the shunt capacitors/reactors and the transformers tap-settings:

$\mathrm{u}=\left(\mathrm{V}_{\mathrm{g}}, \mathrm{T}, \mathrm{Q}_{\mathrm{c}}\right)^{\mathrm{T}}$

or

$\mathrm{u}=\left(\mathrm{V}_{\mathrm{g} 1}, \ldots, \mathrm{V}_{\mathrm{gng}}, \mathrm{T}_{1}, \ldots, \mathrm{T}_{\mathrm{Nt}}, \mathrm{Q}_{\mathrm{c} 1}, \ldots, \mathrm{Q}_{\mathrm{cNc}}\right)^{\mathrm{T}}$

Where ng, nt and nc are the number of generators, number of tap transformers and the number of shunt compensators respectively. 


\section{Objective Function}

\subsection{Active Power Loss}

The objective of the reactive power dispatch is to minimize the active power loss in the transmission network, which can be described as follows:

$F=P L=\sum_{k \in N b r} g_{k}\left(V_{i}^{2}+V_{j}^{2}-2 V_{i} V_{j} \cos \theta_{i j}\right)$

Or

$F=P L=\sum_{i \in N g} P_{g i}-P_{d}=P_{g s l a c k}+\sum_{i \neq s l a c k}^{N g} P_{g i}-P_{d}$

where $g_{k}$ : is the conductance of branch between nodes $\mathrm{i}$ and $\mathrm{j}$, Nbr: is the total number of transmission lines in power systems. $\mathrm{P}_{\mathrm{d}}$ : is the total active power demand, $\mathrm{P}_{\mathrm{gi}}$ : is the generator active power of unit $i$, and $\mathrm{P}_{\text {gsalck: }}$ is the generator active power of slack bus.

\subsection{Voltage Profile Improvement}

For minimizing the voltage deviation in PQ buses, the objective function becomes:

$F=P L+\omega_{v} \times V D$

Where $\omega_{\mathrm{v}}$ : is a weighting factor of voltage deviation.

$\mathrm{VD}$ is the voltage deviation given by:

$V D=\sum_{i=1}^{N p q}\left|V_{i}-1\right|$

\subsection{Equality Constraint}

The equality constraint $\mathrm{g}(\mathrm{x}, \mathrm{u})$ of the ORPD problem is represented by the power balance equation, where the total power generation must cover the total power demand and the power losses:

$P_{G}=P_{D}+P_{L}$

This equation is solved by running Newton Raphson load flow method, by calculating the active power of slack bus to determine active power loss.

\subsection{Inequality Constraints}

The inequality constraints $\mathrm{h}(\mathrm{x}, \mathrm{u})$ reflect the limits on components in the power system as well as the limits created to ensure system security. Upper and lower bounds on the active power of slack bus, and reactive power of generators: 
$P_{\text {gslack }}^{\min } \leq P_{\text {gslack }} \leq P_{\text {gslack }}^{\max }$

$Q_{g i}^{\min } \leq Q_{g i} \leq Q_{g i}^{\max }, i \in N_{g}$

Upper and lower bounds on the bus voltage magnitudes:

$V_{i}^{\min } \leq V_{i} \leq V_{i}^{\max }, i \in N$

Upper and lower bounds on the transformers tap ratios:

$T_{i}^{\min } \leq T_{i} \leq T_{i}^{\max }, i \in N_{T}$

Upper and lower bounds on the compensators reactive powers:

$Q_{c}^{\min } \leq Q_{c} \leq Q_{C}^{\max }, i \in N_{C}$

Where $\mathrm{N}$ is the total number of buses, $\mathrm{N}_{\mathrm{T}}$ is the total number of Transformers; $\mathrm{N}_{\mathrm{c}}$ is the total number of shunt reactive compensators.

\section{Harmony Search Algorithm}

Harmony search (HS) is a relatively new population-based metaheuristic optimization algorithm, that imitates the music improvisation process where the musicians improvise their instruments' pitch by searching for a perfect state of harmony. It was able to attract many researchers to develop HS imitates the natural phenomenon of musicians' behavior when they cooperate the pitches of their instruments together to achieve a fantastic harmony as measured by aesthetic standards. This musicians' prolonged and intense process led them to the perfect state. It is a very successful metaheuristic algorithm that can explore the search space of a given data in parallel optimization environment, where each solution (harmony) vector is generated by intelligently exploring and exploiting a search space. It has many features that make it as a preferable technique not only as standalone algorithm but also to be combined with other metaheuristic algorithms.

The analogy between improvisation and optimization is likely as follows,

1) Each musician corresponds to each decision variable;

2) Musical instrument's pitch range corresponds to the decision variable's value range;

3) Musical harmony at a certain time corresponds to the solution vector at certain iteration;

4) Audience's aesthetics corresponds to the objective function.

Just like musical harmony is improved time after time, solution vector is improved iteration by iteration. In general, HS has five steps and they are described as in Geem as follow:

The optimization problem is defined as follow:

minimizelmaximize $f(a)$,

Subject to $a_{i} \in \mathbf{A}_{i}, i=1,2, \ldots, N$ 
where $f(a)$ is an objective function; a is the set of each decision variable $\left(a_{i}\right)$; $A i$ is the set of possible range of values for each decision variable, $L^{a i} \leq \mathbf{A} i \geq \mathrm{U}^{\text {ai }}$; and $N$ is the number of decision variables.

Then, the parameters of the HS are initialized. These parameters are:

1) Harmony Memory Size (HMS) (i.e. number of solution vectors in harmony memory);

2) Harmony Memory considering Rate (HMCR), where HMCR $\in[0,1]$;

3) Pitch Adjusting Rate (PAR),

Where PAR $\in[0,1]$;

\subsection{Stopping Criteria (i.e. number of improvisation (NI))}

\section{Initialize Harmony Memory}

The harmony memory (HM) is a matrix of solutions with a size of HMS, where each harmony memory vector represents one solution as can be seen in Eq. 3. In this step, the solutions are randomly constructed and rearranged in a reversed order to HM, based on their objective function values such as

$$
\begin{aligned}
& \mathrm{f}\left(\mathrm{a}^{1}\right) \leq \mathrm{f}\left(\mathrm{a}^{2}\right) \\
& \mathrm{HM}=\left[\begin{array}{ccc|c} 
& \ldots . . \leq \mathrm{f}\left(\mathrm{a}^{\mathrm{HMS})} .\right. \\
a_{1}^{1} & \cdots & a_{N}^{1} & f\left(a^{1}\right) \\
\vdots & \ddots & \vdots & \cdot \\
a_{1}^{H M S} & \cdots & a_{N}^{H M S} & \cdot \\
& & & f\left(a^{H M S}\right)
\end{array}\right]
\end{aligned}
$$

This step is the essence of the HS algorithm and the cornerstone that has been building this algorithm. In this step, the HS generates (improvises) a new harmony vector,

$a^{\prime}=\left(a_{1}^{\prime} a_{2}^{\prime} \ldots . . a_{N}^{\prime}\right)$. It is based on three operators: memory consideration; pitch adjustment; or random consideration. In the memory consideration, the values of the new harmony vector are randomly inherited from the historical values stored in HM with a probability of HMCR.

Therefore, the value of decision variable $\left(a_{1}^{\prime}\right)$ is chosen from $\left(a_{1}^{1}, a_{1}^{2}, \ldots \ldots a_{1}^{H M S}\right)$ that is $\left(a_{2}^{\prime}\right)$ is chosen from $\left(a_{2}^{1}, a_{2}^{2}, \ldots \ldots a_{2}^{H M S}\right)$ and the other decision variables, $\left(a_{3}^{\prime}, a_{4}^{\prime}, a_{5}^{\prime}, \ldots ..\right)$, are chosen consecutively in the same manner with the probability of HMCR $\in[0,1]$. The usage of HM is similar to the step where the musician uses his or her memory to generate an excellent tune. This cumulative step ensures that good harmonies are considered as the elements of New Harmony vectors. Out of that, where the other decision variable values are not chosen from HM, according to the HMCR probability test, they are randomly chosen according to their possible range, $\mathrm{a}_{1}^{\prime} \in$ Ai . This case is referred to as random consideration (with a probability of (1-HMCR)), which increases the diversity of the solutions and drives the system further to explore various diverse 
solutions so that global optimality can be attained. The following equation summarized these two steps i.e. memory consideration and random consideration.

$$
a_{i}^{\prime}\left\{\begin{array}{c}
a_{i}^{\prime} \in\left\{a_{i}^{1}, a_{i}^{2}, \ldots \ldots . a_{i}^{H M S}\right\} w . p . H M C R \\
a_{i}^{\prime} \in A_{i} \quad \text { w.p. }(1-H M C R)
\end{array}\right.
$$

Furthermore, the additional search for good solutions in the search space is achieved through tuning each decision variable in the new harmony vector, $a^{\prime}=\left(a_{1}^{\prime} a_{2}^{\prime} \ldots \ldots a_{N}^{\prime}\right)$ inherited from HM using PAR operator. These decision variables $\left(a_{i}^{\prime}\right)$ are examined and to be tuned with the probability of $\operatorname{PAR} \in[0,1]$.

$$
a_{i}^{\prime} \leftarrow\left\{\begin{array}{l}
\text { Adjusting Pitch } w \cdot p \cdot \text { PAR } \\
\text { Doing Nothing } w \cdot p \cdot(1-\text { PAR })
\end{array}\right.
$$

If a generated random number rnd $\in[0,1]$ within the probability of PAR then, the new decision variable $\left(\mathrm{a}_{\mathrm{i}}^{\prime}\right)$ will be adjusted based on the following equation:

$\left(a_{i}^{\prime}\right)=\left(a_{i}^{\prime}\right) \pm \operatorname{rand}() * \mathrm{bw}$

Here, bw is an arbitrary distance bandwidth used to improve the performance of HS and (rand()) is a function that generates a random number $\in[0,1]$. Actually, bw determines the amount of movement or changes that may have occurred to the components of the new vector. The value of bw is based on the optimization problem itself i.e. continuous or discrete. In general, the way that the parameter (PAR) modifies the components of the new harmony vector is an analogy to the musicians' behavior when they slightly change their tone frequencies in order to get much better harmonies. Consequently, it explores more solutions in the search space and improves the searching abilities.

\section{Update the Harmony Memory}

In order to update HM with the new generated vector $a^{\prime}=\left(a_{1}^{\prime}, a_{2}^{\prime}, \ldots \ldots a_{N}^{\prime}\right)$, the objective function is calculated for each New Harmony vector $\mathrm{f}\left(\mathrm{a}^{\prime}\right)$. If the objective function value for the new vector is better than the worst harmony vector stored in HM, then the worst harmony vector is replaced by the new vector. Otherwise, this new vector is ignored.

$a^{\prime} \in H M \wedge a^{\text {worst }} \notin H M$

However, for the diversity of harmonies in HM, other harmonies (in terms of least-similarity) can be considered. Also, the maximum number of identical harmonies in HM can be considered in order to prevent premature HM.

C. Check the stopping criterion 
The iteration process in steps $3 \& 4$ is terminated when the maximum number of improvisations (NI) is reached. Finally, the best harmony memory vector is selected and is considered to be the best solution to the problem under investigation.

\section{Harmony Search Characteristics}

The other important strengths of HS are their improvisation operators, memory consideration; pitch adjustment; and random consideration, that play a major rule in achieving the desired balance between the two major extremes for any optimization algorithm, Intensification and diversification . Essentially, both pitch adjustment and random consideration are the key components of achieving the desired diversification in HS. In random consideration, the new vector's components are generated at random mode, has the same level of efficiency as in other algorithms that handle randomization, where this property allows HS to explore new regions that may not have been visited in the search space. While, the pitch adjustment adds a new way for HS to enhance its diversification ability by tuning the new vector's component within a given bandwidth. A small random amount is added to or subtracted from an existing component stored in HM. This operator, pitch adjustment, is a fine-tuning process of local solutions that ensures that good local solutions are retained, while it adds a new room for exploring new solutions. Further to that, pitch adjustment operator can also be considered as a mechanism to support the intensification of HS through controlling the probability of PAR. The intensification in the HS algorithm is represented by the third HS operator, memory consideration. A high harmony acceptance rate means that good solutions from the history/memory are more likely to be selected or inherited. This is equivalent to a certain degree of elitism. Obviously, if the acceptance rate is too low, solutions will converge more slowly.

\section{Variants of Harmony Search}

Harmony search algorithm got the attention of many researchers to solve many optimization problems such as engineering and computer science problems. Consequently, the interest in this algorithm led the researchers to improve and develop its performance in line with the requirements of problems that are solved. These improvements primarily cover two aspects: (1) improvement of HS in term of parameters setting, and (2) improvements in term of hybridizing of HS components with other metaheuristic algorithms. This section will highlight these developments and improvements to this algorithm in the ten years of this algorithm's age. The first part introduces the improvement of HS in term of parameters setting, while the second part introduces the development of HS in term of hybridizing of HS with other metaheuristic algorithms.

\section{Variants Based on Parameters Setting}

The proper selection of HS parameter values is considered as one of the challenging task not only for HS algorithm but also for other metaheuristic algorithms. This difficulty is a result of different reasons, and the most important one is the absence of general rules governing this aspect. Actually, setting these values is problem dependant and therefore the experimental trials are the only guide to the best values. However, this matter guides the research into new variants of HS. These variants are based on adding some extra components or concepts to make part of these parameters dynamically adapted. The proposed algorithm includes dynamic adaptation for both pitch adjustment rate (PAR) and bandwidth (bw) values. The PAR value is linearly increased in each iteration of HS by using the following equation: 
$\operatorname{PAR}(\mathrm{gn})=\mathrm{PAR}_{\min }+\frac{P A R_{\max }-P A R_{\min }}{N I} \times g n$

Where PAR (gn) is the PAR value for each generation, $\mathrm{PAR}_{\min }$ and $\mathrm{PAR}_{\max }$ are the minimum pitch adjusting rate and maximum pitch adjusting rate respectively. NI is the maximum number of iterations (improvisation) and gn is the generation number. The bandwidth (bw) value is exponentially decreased in each iteration of HS by using the following equation:

$\mathrm{bw}(\mathrm{gn})=\mathrm{bw} \min +\frac{b w_{\max }-b w_{\min }}{N I} \times g n$

where $\mathrm{bw}(\mathrm{gn})$ is the bandwidth value for each generation, $\mathrm{bw}_{\max }$ is the maximum bandwidth, bw $\mathrm{min}_{\text {m }}$ is the minimum bandwidth and gn is the generation number.

\section{Simulation Results}

Harmony Search algorithm (HS) has been tested in practical 191 test system and the following results has been obtained. In Practical 191 test bus system - Number of Generators $=20$, Number of lines $=200$, Number of buses $=191$ Number of transmission lines $=55$. Table 1 shows the optimal control values of practical 191 test system obtained by HS method. And table 2 shows the results about the value of the real power loss by obtained by HS algorithm.

Table 1: Optimal Control values of Practical 191 utility (Indian) system by HS method

\begin{tabular}{|c|c|c|c|}
\hline VG1 & 1.1000 & VG 11 & 0.9000 \\
\hline VG 2 & 0.7000 & VG 12 & 1.0000 \\
\hline VG 3 & 1.0100 & VG 13 & 1.0000 \\
\hline VG 4 & 1.0100 & VG 14 & 0.9000 \\
\hline VG 5 & 1.1000 & VG 15 & 1.0000 \\
\hline VG 6 & 1.1000 & VG 16 & 1.0000 \\
\hline VG 7 & 1.1000 & VG 17 & 0.9000 \\
\hline VG 8 & 1.0100 & VG 18 & 1.0000 \\
\hline VG 9 & 1.1000 & VG 19 & 1.1000 \\
\hline VG 10 & 1.0100 & VG 20 & 1.1000 \\
\hline
\end{tabular}

\begin{tabular}{|c|c|c|c|c|c|}
\hline $\mathrm{T} 1$ & 1.0000 & T21 & 0.9000 & T41 & 0.9000 \\
\hline $\mathrm{T} 2$ & 1.0000 & T22 & 0.9000 & T42 & 0.9000 \\
\hline T3 & 1.0000 & $\mathrm{~T} 23$ & 0.9000 & $\mathrm{~T} 43$ & 0.9100 \\
\hline $\mathrm{T} 4$ & 1.1000 & $\mathrm{~T} 24$ & 0.9000 & $\mathrm{~T} 44$ & 0.9100 \\
\hline T5 & 1.0000 & $\mathrm{~T} 25$ & 0.9000 & $\mathrm{~T} 45$ & 0.9100 \\
\hline T6 & 1.0000 & T26 & 1.0000 & T46 & 0.9000 \\
\hline $\mathrm{T} 7$ & 1.0000 & T27 & 0.9000 & T47 & 0.9100 \\
\hline $\mathrm{T} 8$ & 1.0100 & $\mathrm{~T} 28$ & 0.9000 & $\mathrm{~T} 48$ & 1.0000 \\
\hline T9 & 1.0000 & T29 & 1.0100 & T49 & 0.9000 \\
\hline $\mathrm{T} 10$ & 1.0000 & T30 & 0.9000 & T50 & 0.9000 \\
\hline $\mathrm{T} 11$ & 0.9000 & $\mathrm{~T} 31$ & 0.9000 & T51 & 0.9000 \\
\hline T12 & 1.0000 & $\mathrm{~T} 32$ & 0.9000 & T52 & 0.9000 \\
\hline
\end{tabular}




\begin{tabular}{|l|l|l|l|l|l|l|l|}
\hline T13 & 1.0100 & & T33 & 1.0100 & & T53 & 1.0000 \\
\cline { 1 - 2 } T14 & 1.0100 & & T34 & 0.9000 & T54 & 0.9000 \\
\cline { 1 - 2 } T15 & 1.0100 & T35 & 0.9000 & T55 & 0.9000 \\
\cline { 1 - 2 } T19 & 1.0200 & T39 & 0.9000 & & \\
\cline { 1 - 2 } T20 & 1.0100 & & T40 & 0.9000 & & \\
\hline
\end{tabular}

Table 2: Optimum real power loss values obtained for practical 191 utility (Indian) system by

\begin{tabular}{|l|l|}
\multicolumn{2}{|c}{ HS method. } \\
\hline Real power Loss (MW) & HS \\
\hline Min & 141.4690 \\
\hline Max & 145.2342 \\
\hline Average & 143.0086 \\
\hline
\end{tabular}

\section{Conclusion}

This paper successfully solved the reactive power problem. Harmony search (HS) algorithm emulates the music improvisation progression where musicians improvise their instruments' pitch by searching for a perfect state of harmony. In order to evaluate the efficiency of the proposed algorithm, it has been tested on practical 191 test system.

\section{References}

[1] O. Alsac, B. Scott, "Optimal load flow with steady state security”, IEEE Transaction. PAS -1973, pp. 745-751.

[2] Lee K Y, Paru Y M, Oritz J L -A united approach to optimal real and reactive power dispatch, IEEE Transactions on power Apparatus and systems 1985: PAS-104: 1147-1153

[3] A. Monticelli, M. V.F Pereira, and S. Granville, "Security constrained optimal power flow with post contingency corrective rescheduling", IEEE Transactions on Power Systems :PWRS-2, No. 1, pp.175-182.,1987.

[4] Deeb N, Shahidehpur S.M, Linear reactive power optimization in a large power network using the decomposition approach. IEEE Transactions on power system 1990: 5(2): 428-435

[5] E. Hobson,' Network consrained reactive power control using linear programming, 'IEEE Transactions on power systems PAS -99 (4), pp 868=877, 1980

[6] K.Y Lee, Y.M Park, and J.L Oritz, "Fuel -cost optimization for both real and reactive power dispatches", IEE Proc; 131C, (3), pp.85-93.

[7] M.K. Mangoli, and K.Y. Lee, "Optimal real and reactive power control using linear programming", Electr. Power Syst. Res, Vol.26, pp.1-10,1993.

[8] C.A. Canizares, A.C.Z.de Souza and V.H. Quintana, "Comparison of performance indices for detection of proximity to voltage collapse," vol. 11. no.3, pp.1441-1450, Aug 1996.

[9] K. Anburaja, "Optimal power flow using refined genetic algorithm", Electr. Power Compon. Syst, Vol. 30, 1055-1063,2002.

[10] D. Devaraj, and B. Yeganarayana, "Genetic algorithm based optimal power flow for security enhancement", IEE proc-Generation. Transmission and. Distribution; 152, 6 November 2005.

[11] A. Berizzi, C. Bovo, M. Merlo, and M. Delfanti, "A ga approach to compare orpf objective functions including secondary voltage regulation," Electric Power Systems Research, vol. 84, no. 1, pp. $187-194,2012$. 
[12] C.-F. Yang, G. G. Lai, C.-H. Lee, C.-T. Su, and G. W. Chang, "Optimal setting of reactive compensation devices with an improved voltage stability index for voltage stability enhancement," International Journal of Electrical Power and Energy Systems, vol. 37, no. 1, pp. 50 - 57, 2012.

[13] P. Roy, S. Ghoshal, and S. Thakur, "Optimal var control for improvements in voltage profiles and for real power loss minimization using biogeography-based optimization," International Journal of Electrical Power and Energy Systems, vol. 43, no. 1, pp. 830 - 838, 2012.

[14] B. Venkatesh, G. Sadasivam, and M. Khan, "A new optimal reactive power scheduling method for loss minimization and voltage stability margin maximization using successive multi-objective fuzzy lp technique," IEEE Transactions on Power Systems, vol. 15, no. 2, pp. 844 - 851, may 2000.

[15] W. Yan, S. Lu, and D. Yu, "A novel optimal reactive power dispatch method based on an improved hybrid evolutionary programming technique," IEEE Transactions on Power Systems, vol. 19, no. 2, pp. 913 - 918, may 2004.

[16] W. Yan, F. Liu, C. Chung, and K. Wong, "A hybrid genetic algorithminterior point method for optimal reactive power flow," IEEE Transactions on Power Systems, vol. 21, no. 3, pp. 1163 1169, aug. 2006.

[17] J. Yu, W. Yan, W. Li, C. Chung, and K. Wong, "An unfixed piecewiseoptimal reactive power-flow model and its algorithm for ac-dc systems," IEEE Transactions on Power Systems, vol. 23, no. 1, pp. $170-176$, feb. 2008.

[18] F. Capitanescu, "Assessing reactive power reserves with respect to operating constraints and voltage stability," IEEE Transactions on Power Systems, vol. 26, no. 4, pp. 2224-2234, nov. 2011.

[19] Z. Hu, X. Wang, and G. Taylor, "Stochastic optimal reactive power dispatch: Formulation and solution method," International Journal of Electrical Power and Energy Systems, vol. 32, no. 6, pp. $615-621,2010$.

[20] Kargarian, M. Raoofat, and M. Mohammadi, "Probabilistic reactive power procurement in hybrid electricity markets with uncertain loads," Electric Power Systems Research, vol. 82, no. 1, pp. 68 $-80,2012$.

[21] Gao XZ, Wang X, Ovaska SJ, "Modified harmony search methods for uni-modal and multi-modal optimization", In: Proceedings of the 2008 8th international conference on hybrid intelligent systems. Pp 65-72. (2008)

[22] Gao XZ,Wang X, Ovaska SJ, "Uni-modal and multi-modal optimization using modified harmony search methods", Int J Innov Comput Inf Control. (2009)

[23] Geem Z, "Improved harmony search from ensemble of music players", In: Gabrys B, Howlett RJ, Jain L (eds) Knowledge-based intelligent information and engineering systems. Springer, Heidelberg, pp 86-93. (2006)

[24] Geem Z, "Optimal scheduling of multiple dam system using harmony search algorithm", In: Computational and ambient intelligence. Springer, Berlin, pp 316-323. (2007)

\footnotetext{
*Corresponding author.

E-mail address: gklenin@ gmail.com
} 